\title{
Herz-Lungen-Risiko durch COPD-Medis erhöht?
}

Hintergrund und Fragestellung: Lang wirksame Bronchodilatatoren (LABA) und lang wirksame Anticholinergika (LAMA) werden heute als Erstlinientherapie bei COPD empfohlen. Beide Substanzgruppen haben potenzielle kardiale Nebenwirkungen, vor allem Tachyarrhythmien und die Verschlechterung einer koronaren Herzkrankheit. Die bisher vorliegenden randomisierten Studien und Beobachtungsstudien erbrachten keine klare Antwort zum jeweiligen kardialen Risiko, das sich möglicherweise bereits kurz nach Therapiebeginn manifestieren könnte. Dem sollte mit der aktuellen Studie Klarheit folgen.

Patienten und Methoden: Die Clinical Practice Research Data Link ist eine Datenbank in UK, welche die medizinischen Daten von mehr als 10 Millionen Personen enthält, die in über 600 allgemeinärztlichen Praxen in England behandelt werden. Sämtliche medizinischen Maßnahmen und Medikamenten-Verordnungen und stationäre Behandlungen können über die Verlinkung verschiedener Datenbanken in der Zusammenschau ausgewertet werden. Die Untersuchung bezieht sich auf alle Patienten im Alter über 55 Jahre, die zwischen 1. Januar 2001 (das Jahr, ehe Tiotropium in den englischen Markt eingeführt wurde) und 31. August 2012

Originalie

Suissa S, Dell'Aniello S, Ernst P. Long-Acting Bronchodilator Initiation in COPD and the Risk of Adverse Cardiopulmonary Events: A Population-Based Comparative Safety Study. Chest. 2017 Jan;151(1):60-7. entweder einen LABA oder Tiotropium verordnet bekamen. Alle Patienten mussten eine mindestens zwei Jahre bestehende entsprechende Vorgeschichte für COPD aufweisen. Patienten, die beide Substanzen zum selben Datum verordnet bekamen, wurden ausgeschlossen. Um zu vermeiden, dass schwerer kranke Patienten überproportional in die Untersuchung aufgenommen wurden (die die neuen Substanzen mit größerer Wahrscheinlichkeit verordnet bekommen) wurden nur Personen in die Studie aufgenommen, deren erste Verordnung von Tiotropium mindestens ein Jahr nach der Markteinführung erfolgte. Die gesamte Kohorte wurde ein Jahr lang mit allen zwischenzeitlich aufgetretenen medizinischen Problemen verfolgt. Die Studie wurde beendet ein Jahr nach Aufnahme, zum Zeitpunkt des Todes, am 31. August 2013 oder bei nicht mehr gelieferten Daten aus der Praxis, je nachdem, welches Ereignis zuerst eintrat.

Ergebnisse: 26.442 Patienten mit Beginn einer Tiotropium-Therapie wurden verglichen mit 26.442 Personen mit Beginn einer LABA-Therapie, überwiegend kombiniert mit inhalativen Kortikosteroiden. Die Hazard Ratio eines akuten Myokardinfarkts in Assoziation mit dem Beginn der Tiotropium-Therapie im Vergleich zum Beginn mit LABA betrug 1,10, für Schlaganfall 1,02, für Arrhythmien 0,81 und für Herzinsuffizienz 0,90. Alle diese Unterschiede waren statistisch nicht signifikant. Die Inzidenz von Pneumonien war unter Tiotropium signifikant geringer (HR: 0,81) als unter LABA. Während Patienten mit Tiotropium überwiegend als Monotherapie behandelt wurden, hatten $78 \%$ der LABA-Anwender eine Kombination aus LABA und inhalativen Steroiden verordnet bekommen.

Schlussfolgerung: Die neu begonnene Therapie bei COPD mit Tiotropium erhöht im ersten Behandlungsjahr im Vergleich zu den LABA nicht das kardiale Risiko. LABA gehen in dieser Studie jedoch mit einer höheren Pneumonierate einher, die aber wahrscheinlich auf die meist damit kombinierten Steroide zurückzuführen ist.

\section{- Kommentar von Prof. Dr. med. Hermann S. Füeßl}

\section{Eigentlich sind die Ergebnisse schon überholt}

Die Studie überzeugt durch den eleganten methodischen Ansatz und enorme Patientenzahlen, wie es nur in einem Land mit umfassender elektronischer Dokumentation des gesamten medizinischen Versorgungssystems möglich ist. Durch entsprechende Verlinkung von Datenbanken gelingt vor allem eine gemeinsame Betrachtung von Medizin in Praxen und im Krankenhaus. Damit gelingt es in relativ kurzer Zeit, Auswirkungen einer neuen Therapie und seltene unerwünschte Wirkungen unter den Bedingungen des medizinischen Alltags zu finden, wie es in einer kontrollierten Studie nie möglich ist. Von derartigen Daten können wir in Deutschland nur träumen. Natürlich haben auch derartige Studien ihre Limitationen, vor allem kann aus ihnen die Compliance nicht abgelesen werden. Angesichts der raschen Entwicklung der Therapie der COPD erscheint die Studie, obwohl 2017 publiziert, aber schon veraltet, da die hier beantworteten Fragen in den Leitlinien nur noch eine untergeordnete Rolle spielen. Besonders die Bedeutung der inhalativen Steroide hat jüngst stark abgenommen. Damit könnte das Pneumonierisiko gesunken sein. Auch sind LABA-LAMA-Kombinationen auf den Markt gekommen.

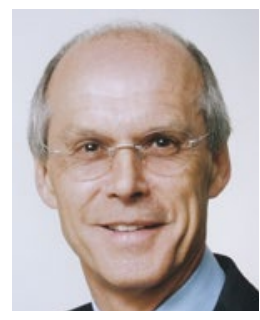

Prof. Dr. med. Hermann S. FüeßI

Internist - Gastroenterologe

Privatpraxis für Integrative Innere Medizin

Renatastr. 50, 80639 München

hsfuessl@t-online.de 